\title{
Fock Space Structure for the Simplest Parasupersymmetric System 娄
}

\author{
Weimin Yang and Sicong Jing \\ Department of Modern Physics, University of Science and \\ Technology of China, Hefei 230026, P.R. China
}

November 23, 2018

\begin{abstract}
Structure of the state-vector space for a system consisting of one mode parabose and one mode parafermi degree of freedom with the same parastatistics order $p$ is studied and a complete, orthonormal set of basis vectors in this space is constructed. There is an intrinsic double degeneracy for state vectors with $m$ parabosons and $n$ parafermions, where $m \neq 0, n \neq 0$, and $n \neq p$. It is also shown that the degeneracy plays a key role in realization of exact supersymmetry for such a system.
\end{abstract}

\section{Introduction}

One of the fundamental and powerful approaches in theoretical physics is a search for symmetries. Generalized statistics first was introduced by Green in the form of parastatistics as an exotic possibility extending the Bose and Fermi statistics [1]. Recent research reveals that it probably has some potential applications in the physics of the quantum Hall effect and the high temperature superconductivity [2]. Supersymmetry, on the other hand, unifies Bose and Fermi statistics and its development leads to important progress in field and string theories [3].

A natural attempt is to construct a supersymmetric theory which can unify parabose and parafermi statistics. Though some authors generalized the supersymmetric quan-

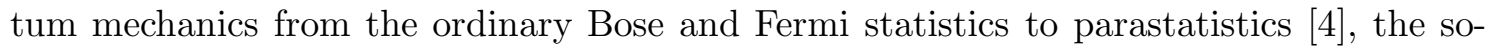
called parasupersymmetric quantum mechanics actually only unified ordinary bosons and parafermions, or ordinary fermions and parabosons [5]. In other words, these works only unified parabosons and parafermions with different parastatistics order $p$. It is well known

\footnotetext{
*This project supported by National Science Foundation of China and LWTZ 1298.
} 
from the parastatistics that if the parabosons and parafermions are with different order $p$, they will commute or anticommute with each other according to their algebraic relations being relative parabose or relative parafermi, respectively [6]. In these cases the Fock space structures of the parasupersymmetric systems are relatively simple. However, if the parabosons and parafermions are with a same order $p$, they will neither commute nor anticommute with each other and will satisfy some complicated trilinear commutation relations. The Fock space structures for these systems are still unknown yet, so far it is not really available to construct a supersymetric quantum mechanics theory for parabosons and parafermions with the same order $p$.

In this paper, we first study the Fock space structure for a system with one mode parabose and one mode parafermi degree of freedom which are with the same order $p$, and construct a complete, orthonormal set of basis vectors for this parasystem. Due to the complexity of the parastatistics, there is an intrinsic double degeneracy of state vectors in this Fock space. Based on this understanding to the Fock space structure, we build a parasupersymmetric quantum mechanics model for the same order $p$ parabosons and parafermions, and the degeneracy plays an important role in the building.

\section{Fock space structure}

It is well known that for one mode parabose system, the fundamental trilinear relations are

$$
\left[a,\left\{a^{\dagger}, a\right\}\right]=2 a, \quad\left[a, a^{\dagger 2}\right]=2 a^{\dagger}, \quad\left[a, a^{2}\right]=0,
$$

where $a^{\dagger}$ and $a$ are parabose creation and annihilation operator respectively, and for one mode parafermi system, the fundamental relation is

$$
\left[f,\left[f^{\dagger}, f\right]\right]=2 f
$$

where $f^{\dagger}$ and $f$ are parafermi creation and annihilation operator respectively. Moreover, for a system consisting of one parabose and one parafermi degree of freedom with a same order $p$, besides the relations (1) and (2), there are the following mixed trilinear relations [7]

$$
\begin{aligned}
& {\left[f,\left\{a^{\dagger}, a\right\}\right]=0,\left[a,\left[f^{\dagger}, f\right]\right]=0,\left[f, a^{2}\right]=0,\left[f^{\dagger}, a^{2}\right]=0,} \\
& {\left[a,\left\{f^{\dagger}, a\right\}\right]=0,[a,\{f, a\}]=0,\{f,\{f, a\}\}=0,\left\{f,\left\{a^{\dagger}, f\right\}\right\}=0,} \\
& {\left[a,\left\{f, a^{\dagger}\right\}\right]=2 f,\left[a^{\dagger},\{a, f\}\right]=-2 f,\left\{f,\left\{f^{\dagger}, a\right\}\right\}=2 a,\left\{f^{\dagger},\{a, f\}\right\}=2 a,}
\end{aligned}
$$

together with the adjoint ones. From (3) we see that one paraboson does neither commute nor anticommute with parafermions, however, two parabosons do commute with parafermions. According to parastatistics [8], in order to study the structure of a state 
vector space, we should impose the following unique vacuum state conditions

$$
\begin{aligned}
& a|0\rangle=0, f|0\rangle=0, a f^{\dagger}|0\rangle=0, f a^{\dagger}|0\rangle=0, \\
& a a^{\dagger}|0\rangle=p|0\rangle, \quad f f^{\dagger}|0\rangle=p|0\rangle
\end{aligned}
$$

Let us denote the parabose and the parafermi number operator as $N_{a}$ and $N_{f}$ respectively,

$$
N_{a}=\frac{1}{2}\left\{a^{\dagger}, a\right\}-\frac{p}{2}, \quad N_{f}=\frac{1}{2}\left[f^{\dagger}, f\right]+\frac{p}{2} .
$$

Obviously, $N_{a}$ commutes with $N_{f}$, and the vacuum state $|0\rangle$ is their common eigenstate with the eigenvalue zero: $N_{a}|0\rangle=0$ and $N_{f}|0\rangle=0$. For the sake of later convenience, we introduce four useful operators $N_{s}, T, Q_{s}$ and $Q_{s}^{\dagger}$ which are defined by

$$
\begin{aligned}
& N_{s}=\frac{1}{p}\left(N_{f}^{2}-(p+1) N_{f}+f^{\dagger} f+\frac{p}{2}\right), \\
& T=\frac{p}{2}\left(F^{\dagger} F+Q^{\dagger} Q-N_{a}-\frac{p}{2}\right)-2\left(N_{a}+\frac{p}{2}\right)\left(N_{f}-\frac{p}{2}\right) N_{s}, \\
& Q_{s}=\left(\frac{1}{2}-N_{s}\right) T, \quad Q_{s}^{\dagger}=\left(\frac{1}{2}+N_{s}\right) T,
\end{aligned}
$$

where $F=\frac{1}{2}\{a, f\}$ and $Q=\frac{1}{2}\left\{a^{\dagger}, f\right\}$. It is readily to know the following relations between these operators

$$
\begin{aligned}
& {\left[N_{a}, N_{s}\right]=0,\left[N_{f}, N_{s}\right]=0,\left[N_{s}, f\right]=0,\left[N_{s}, a^{2}\right]=0,} \\
& {\left[N_{a}, T\right]=0,\left[N_{f}, T\right]=0,\left[N_{a}, Q_{s}\right]=0,\left[N_{f}, Q_{s}\right]=0,} \\
& \left\{N_{s}, T\right\}=0,\left[N_{s}, Q_{s}\right]=-Q_{s},\left[Q_{s}^{\dagger}, Q_{s}\right]=2 T^{2} N_{s},\left\{Q_{s}^{\dagger}, Q_{s}\right\}=T^{2} .
\end{aligned}
$$

We now investigate the state space structure for our parasystem. Firstly, we search common eigenstates of the operators $N_{a}$ and $N_{f}$, which are denoted by $|m, n\rangle$

$$
N_{a}|m, n\rangle=m|m, n\rangle, \quad N_{f}|m, n\rangle=n|m, n\rangle .
$$

Obviously, there are $m$ parabosons and $n$ parafermions in the state $|m, n\rangle$. Using $\left[N_{a}, a^{\dagger n}\right]=$ $n a^{\dagger n},\left[N_{f}, f^{\dagger n}\right]=n f^{\dagger n},\left[N_{a}, f^{\dagger}\right]=0$ and $\left[N_{f}, a^{\dagger}\right]=0$, we have

Lemma 1. A state, produced by $m$ operators $a^{\dagger}$ and $n$ operators $f^{\dagger}$ acting on the vacuum state $|0\rangle$ in any permutation, is the common eigenstate of $N_{a}$ and $N_{f}$.

We can write the state $|m, n\rangle$ as

$$
|m, n\rangle=f^{\dagger n_{0}} a^{\dagger m_{1}} f^{\dagger n_{1}} a^{\dagger m_{2}} f^{\dagger n_{2}} \ldots f^{\dagger n_{l-1}} a^{\dagger m_{l}} f^{\dagger n_{l}}|0\rangle
$$

where $m_{1}+m_{2}+\ldots+m_{l}=m, n_{0}+n_{1}+n_{2}+\ldots+n_{l}=n$, and all the $m_{i} \geq 1,(i=1, \ldots, l)$, all the $n_{i} \geq 1,(i=1, \ldots, l-1)$, with $n_{0}, n_{l} \geq 0$. From the Lemma 1 we see that any two partitions $\left(m_{1}, m_{2}, \ldots, m_{l}\right)$ and $\left(n_{0}, n_{1}, n_{2}, \ldots, n_{l}\right)$ satisfying $\sum_{i=1}^{l} m_{i}=m, \sum_{i=0}^{l} n_{i}=n$ will correspond to a state $|m, n\rangle$. Since $a^{\dagger}$ and $f^{\dagger}$ neither commute nor anticommute with each 
other, it is quite possible that several different permutations of the operators $a^{\dagger}$ and $f^{\dagger}$ correspond to a same state $|m, n\rangle$, or that there is an intrinsic degeneracy in the subspace spanned by different eigenstates of $N_{a}$ and $N_{f}$ with the same eigenvalues $m$ and $n$ (we use the symbol $(\mathrm{m}, \mathrm{n})$ to denote this subspace). In order to make the structure of the subspace $(\mathrm{m}, \mathrm{n})$ clear, we furthermore have

Lemma 2. The subspace $(m, n)$ is usually two dimensional, except for three special cases, i.e., for $(0, n),(m, 0)$ and $(m, p)$, in which the intrinsic degeneracy disappears.

To prove the Lemma 2, let us use $\left[a^{\dagger 2}, f^{\dagger}\right]=0$, and shift all the even powers of $a^{\dagger}$ in the right-hand of (9) to left side of the permutation of $a^{\dagger}$ and $f^{\dagger}$, which will lead to

$$
|m, n\rangle=a^{\dagger(m-s)} f^{\dagger k_{0}} a^{\dagger} f^{\dagger k_{1}} a^{\dagger} f^{\dagger k_{2}} \ldots f^{\dagger k_{s-1}} a^{\dagger} f^{\dagger k_{s}}|0\rangle
$$

where $m-s$ is an even integer, and $k_{0}+k_{1}+k_{2}+\ldots+k_{s}=n$ with $k_{1}, k_{2}, \ldots, k_{s-1} \geq 1$ and $k_{0}, k_{s} \geq 0$. Then repeatedly using the relations $a^{\dagger} f^{\dagger k}=(-)^{k} f^{\dagger k} a^{\dagger}+2 k F^{\dagger} f^{\dagger(k-1)},\left[F^{\dagger}, a^{\dagger}\right]=$ $0,\left\{F^{\dagger}, f^{\dagger}\right\}=0$ and $F^{\dagger 2}=0$, we may rewrite the permutation $a^{\dagger} f^{\dagger k_{1}} a^{\dagger} f^{\dagger k_{2}} \ldots a^{\dagger} f^{\dagger k_{s}}$ as a sum of two terms

$$
a^{\dagger} f^{\dagger k_{1}} a^{\dagger} f^{\dagger k_{2}} \ldots a^{\dagger} f^{\dagger k_{s}}=\alpha_{s} f^{\dagger\left(k_{1}+k_{2}+\ldots+k_{s}\right)} a^{\dagger s}+\beta_{s} f^{\dagger\left(k_{1}+k_{2}+\ldots+k_{s}-1\right)} a^{\dagger(s-1)} F^{\dagger},
$$

where $\alpha_{s}$ and $\beta_{s}$ are two coefficients decided by the integer $s$. For instance, we have

$$
\begin{aligned}
& a^{\dagger} f^{\dagger k_{1}}=(-)^{k_{1}} f^{\dagger k_{1}} a^{\dagger}+(-)^{k_{1}-1} 2 k_{1} f^{\dagger\left(k_{1}-1\right)} F^{\dagger}, \\
& a^{\dagger} f^{\dagger k_{1}} a^{\dagger} f^{\dagger k_{2}}=(-)^{k_{1}} f^{\dagger\left(k_{1}+k_{2}\right)} a^{\dagger 2}+(-)^{k_{1}-1} 2 k_{1} f^{\dagger\left(k_{1}+k_{2}-1\right)} a^{\dagger} F^{\dagger}, \\
& a^{\dagger} f^{\dagger k_{1}} a^{\dagger} f^{\dagger k_{2}} a^{\dagger} f^{\dagger k_{3}}=(-)^{k_{1}+k_{3}} f^{\dagger\left(k_{1}+k_{2}+k_{3}\right)} a^{\dagger 3}+(-)^{k_{2}} 2\left(k_{1}+k_{3}\right) f^{\dagger\left(k_{1}+k_{2}+k_{3}-1\right)} a^{\dagger 2} F^{\dagger},
\end{aligned}
$$

and so on. Substituting (11) into (10), we arrive at

$$
|m, n\rangle=\alpha_{s} f^{\dagger n} a^{\dagger m}|0\rangle+\beta_{s} f^{\dagger(n-1)} a^{\dagger(m-1)} F^{\dagger}|0\rangle,
$$

which means that we can write any state $|m, n\rangle(9)$ satisfying the conditions $\sum_{i=1}^{l} m_{i}=$ $m$ and $\sum_{i=0}^{l} n_{i}=n$ as a linear combination of two basic state-vectors $f^{\dagger n} a^{\dagger m}|0\rangle$ and $f^{\dagger(n-1)} a^{\dagger(m-1)} F^{\dagger}|0\rangle$. It is worth pointing out that for the $n \geq p+1$ case, both the two basic state-vectors will become zero because the parastatistics order is $p$. Thus the state $|m, n\rangle$ will vanish for $n \geq p+1$ case. As for why the degeneracy in the subspace $(\mathrm{m}, \mathrm{n})$ will disappear for three special case (i.e., for $(0, n),(m, 0)$ and $(m, p)$ cases), please see the words following (15). Combining the Lemma 1 and the Lemma 2, we have the following theorem

Theorem. Any common eigenstate $|m, n\rangle$ of the parabose nnumber operator $N_{a}$ and the parafermi number operator $N_{f}$ with the eigenvalues $m$ and $n$ respectively can be expressed as a combination of two basic state-vectors $f^{\dagger n} a^{\dagger m}|0\rangle$ and $f^{\dagger(n-1)} a^{\dagger(m-1)} F^{\dagger}|0\rangle$, as in (12), where $m \neq 0, n \neq 0$ and $n \neq p$.

We now denote the two basic state-vectors as $|m, n, \alpha\rangle=f^{\dagger n} a^{\dagger m}|0\rangle$ and $|m, n, \beta\rangle=$ $f^{\dagger(n-1)} a^{\dagger(m-1)} F^{\dagger}|0\rangle$. After some algebras we see that usually the inner products of the two 
basic state-vectors are not zero, i.e., $\langle m, n, \alpha \mid m, n, \beta\rangle \neq 0$, or the two basic state-vectors are not orthogonal. Using Schmidt method we can get two orthonormal basis vectors in the subspace $(m, n)$

$$
\begin{aligned}
& \left|m, n, s=\frac{1}{2}\right\rangle=\sqrt{\frac{(p-n) !}{p ! n ![m] !}} f^{\dagger n} a^{\dagger m}|0\rangle \\
& \left|m, n, s=-\frac{1}{2}\right\rangle=\sqrt{\frac{(p-n-1) !\left(m+\frac{1-(-)^{m}}{2}\right)}{p !(n-1) ![m+1] !}} f^{\dagger(n-1)}\left(p F^{\dagger}-f^{\dagger} a^{\dagger}\right) a^{\dagger(m-1)}|0\rangle,(13)
\end{aligned}
$$

which satisfy

$$
\left\langle m, n, s \mid m^{\prime}, n^{\prime}, s^{\prime}\right\rangle=\delta_{m, m^{\prime}} \delta_{n, n^{\prime}} \delta_{s, s^{\prime}}
$$

and

$$
\sum_{m=0}^{\infty} \sum_{n=0}^{p} \sum_{s= \pm 1 / 2}|m, n, s\rangle\langle m, n, s|=1,
$$

where $[m]=m+(p-1) \frac{1-(-)^{m}}{2},[m] !=[m][m-1] \ldots[1]$ and $[0] !=1$. Some remarks concerning the basis vector $|m, n, s\rangle$ are as follows. Firstly, for $m=0$ or $n=0$ case, by the construction of the state $|m, n\rangle$, only the state $|m, n, 1 / 2\rangle$ exists, which exactly coincides with the basis vectors of one mode parafermi system or the basis vectors of one mode parabose system respectively. This can also be seen from the normalization factor of the state $|m, n,-1 / 2\rangle$. For example, when $m=0$, the normalization factor of the state $|m, n,-1 / 2\rangle$ is zero, which means the state $|m, n,-1 / 2\rangle$ does not exist. Secondly, for $n=p$ case, using a formula $p f^{\dagger(p-1)} a^{\dagger} f^{\dagger}+(p-2) f^{\dagger p} a^{\dagger}=0$ which works for paraboson and parafermion with the same order $p$, we also see the state $|m, n,-1 / 2\rangle$ vanishes. So in the three special cases $(\mathrm{m}=0, \mathrm{n}=0$, or $\mathrm{n}=\mathrm{p})$, there exist only the states $|m, n, 1 / 2\rangle$, or the intrinsic double degeneracy in the subspace $(m, n)$ disappears. Needless to say, if the order $p=1$, our parasystem will reduce to the ordinary boson and fermion system, and of course the degeneracy will disappear. Also when $n \geq p+1$, all the basis vectors will become zero.

Furthermore, we show that the two basis vectors $|m, n, 1 / 2\rangle$ and $|m, n,-1 / 2\rangle$ are eigenstates of the operator $N_{s}$ defined by (6) with eigenvalue $1 / 2$ and $-1 / 2$ respectively. In fact, noticing $\left[N_{s}, f^{\dagger}\right]=0,\left[N_{s}, a^{\dagger 2}\right]=0$, and $N_{s}|0\rangle=\frac{1}{2}|0\rangle, N_{s} a^{\dagger}|0\rangle=\frac{1}{2} a^{\dagger}|0\rangle$, we have

$$
N_{s}|m, n, 1 / 2\rangle=\frac{1}{2}|m, n, 1 / 2\rangle .
$$

Then using $N_{f} F^{\dagger}|0\rangle=F^{\dagger}|0\rangle$, and $N_{f} F^{\dagger} a^{\dagger}|0\rangle=F^{\dagger} a^{\dagger}|0\rangle$, we have

$$
\begin{aligned}
& N_{s}\left(p F^{\dagger}-f^{\dagger} a^{\dagger}\right)|0\rangle=-\frac{1}{2}\left(p F^{\dagger}-f^{\dagger} a^{\dagger}\right)|0\rangle, \\
& N_{s}\left(p F^{\dagger}-f^{\dagger} a^{\dagger}\right) a^{\dagger}|0\rangle=-\frac{1}{2}\left(p F^{\dagger}-f^{\dagger} a^{\dagger}\right) a^{\dagger}|0\rangle,
\end{aligned}
$$


which will lead to

$$
N_{s}|m, n,-1 / 2\rangle=-\frac{1}{2}|m, n,-1 / 2\rangle .
$$

Thus we clearly understand the Fock space structure of our parasystem. The set of complete and orthonormal basis vectors in this Fork space is $\{|m, n, s\rangle\}$ which are common eigenstates of three operators $N_{a}, N_{f}$ and $N_{s}$.

Before concluding this section, let us see actions of the operators $T, Q_{s}$ and $Q_{s}^{\dagger}$ defined by (6) on the basis states $|m, n, s\rangle$. After some simple calculations we find that

$$
\begin{aligned}
& T|m, n, 1 / 2\rangle=\sqrt{n(p-n)\left(m^{2}+m p+p^{2}\left(1-(-)^{m}\right) / 8\right)}|m, n,-1 / 2\rangle, \\
& T|m, n,-1 / 2\rangle=\sqrt{n(p-n)\left(m^{2}+m p+p^{2}\left(1-(-)^{m}\right) / 8\right)}|m, n, 1 / 2\rangle,
\end{aligned}
$$

which mean that $T$ is a transition operator changing $|m, n, 1 / 2\rangle$ to $|m, n,-1 / 2\rangle$ and vice versa. In addition, we also have

$$
\begin{aligned}
& Q_{s}|m, n, 1 / 2\rangle=\sqrt{n(p-n)\left(m^{2}+m p+p^{2}\left(1-(-)^{m}\right) / 8\right)}|m, n,-1 / 2\rangle, \\
& Q_{s}|m, n,-1 / 2\rangle=0,
\end{aligned}
$$

and

$$
\begin{aligned}
& Q_{s}^{\dagger}|m, n, 1 / 2\rangle=0, \\
& Q_{s}^{\dagger}|m, n,-1 / 2\rangle=\sqrt{n(p-n)\left(m^{2}+m p+p^{2}\left(1-(-)^{m}\right) / 8\right)}|m, n, 1 / 2\rangle,
\end{aligned}
$$

which imply that $Q_{s}$ and $Q_{s}^{\dagger}$ are ladder operators in the two dimensional subspace $(\mathrm{m}, \mathrm{n})$.

\section{Parasupersymmetric quantum mechanics model}

Now we consider the simplest parasupersymmetric model consisting of a free parabose oscillator and a free parafermi oscillator with the Hamiltonian

$$
H=\frac{1}{2}\left\{a^{\dagger}, a\right\}+\frac{1}{2}\left[f^{\dagger}, f\right]=N_{a}+N_{f}
$$

¿From our basic relations in (3), we know that the operators $H, Q$ and $Q_{\dagger}$ satisfy the standard supersymmetric algebra

$$
[H, Q]=\left[H, Q^{\dagger}\right]=0, \quad\left\{Q, Q^{\dagger}\right\}=H, \quad Q^{2}=Q^{\dagger 2}=0,
$$

so $Q$ and $Q^{\dagger}$ are aupersymmetric charge operators which realize the supersymmetry between parabosons and parafermions. It is easily to see that $Q$ (or $Q^{\dagger}$ ) changes states between two subspaces $(\mathrm{m}, \mathrm{n})$ and $(\mathrm{m}+1, \mathrm{n}-1)$ (or $(\mathrm{m}, \mathrm{n})$ and $(\mathrm{m}-1, \mathrm{n}+1))$. In the previous section we construct a set of complete and orthonormal basis vectors $\{|m, n, s\rangle\}$ for 
our parasystem. In this basis the spectrum of the Hamiltonian (22) can be immediately obtained

$$
H|m, n, s\rangle=E_{m+n}|m, n, s\rangle=(m+n)|m, n, s\rangle,
$$

where $m \geq 0$ and $0 \leq n \leq p$. We would like to emphasize here that there is an intrinsic double degeneracy for any state with $m$ parabosons and $n$ parafermions, except for three extreme cases in which $m=0$, or $n=0$, or $n=p$. For $p=3$ case, the spectrum is shown in Fig.1. From the figure we see that the ground state level $E_{0}=0$ is nondegenerate, which results from the assumption of the unique vacuum state and also means that the parasupersymmetry is unbroken. The first level $E_{1}$ is double degenerate, to which there are two states $|1,0,1 / 2\rangle$ and $|0,1,1 / 2\rangle$ corresponding. The second level $E_{2}$ is quadruple degenerate, to which four states $|2,0,1 / 2\rangle,|0,2,1 / 2\rangle,|1,1,1 / 2\rangle$ and $|1,1,-1 / 2\rangle$ correspond. Generally speaking, the degeneracy of the $l$-th level $(l \leq p) E_{l}$ is $2 l$-fold, or there are $2 l$ states with the energy $E_{l}=l$, which are $|l, 0,1 / 2\rangle,|0, l, 1 / 2\rangle$, and $|m, n, \pm 1 / 2\rangle$, where $m=1,2, \ldots, l-1$, so $n=l-1, l-2, \ldots, 1$, respectively. However, for $l>p$ case, the degeneracy of the level $E_{l}$ is the same $2 p$-fold, or there are always $2 p$ states having the energy $E_{l}=l$, which are $|l, 0,1 / 2\rangle,|l-p, p, 1 / 2\rangle$, and $|m, n, \pm 1 / 2\rangle$, where $n=1,2, \ldots, p-1$ and correspodingly $m=l-1, l-2, \ldots, l-p+1$.

Here we find that the intrinsic double degeneracy in the subspace $(m, n)$ plays an important role in realization of the supersymmetry for our parasystem. From the ordinary supersymmetric quantum mechanics we know that the number of bose states is equal to the number of fermi states in the Fock space of the theory [9]. If one introduces the Witten index [10], or an operator $(-)^{N_{F}}$, where $N_{F}$ is the fermi number operator, then one must have $\operatorname{Tr}(-)^{N_{F}}=0$ for a supersymmetric quantum system, here the trace is done for the all physical states except the vacuum state. In our case, we should generalize the Witten index to the form of $(-)^{N_{f}}$, where the $N_{f}$ is the parafermi number operator, and if our parasystem is supersymmetry, we must also have $\operatorname{Tr}(-)^{N_{f}}=0$, here the trace is done for our all states except the vacuum state. In other words, for our parasystem, if the supersymmtry is exactly established, the number of states with even parafermions must exactly be equal to the number of states with odd parafermions. Due to the intrinsic double degeneracy in the subspace $(\mathrm{m}, \mathrm{n})$ (where $m \neq 0, n \neq 0$ and $n \neq p), \operatorname{Tr}(-)^{N_{f}}$ is exact zero, here the trace is done for all states except the vacuum state. To see this, let us consider every excitation level $E_{l}$. In every such level, we have even states no matter $l \leq p$ or $l>p$, and half of them are even- and half of them are odd-parafermion states, so $\operatorname{Tr}(-)^{N_{f}}=0$. If we had without the double degeneracy, we would have no way to realize the supersymmetry between the parabosons and the parafermions. It is exactly in this sense, we say that the intrinsic double degeneracy plays an fundamental rule in building the parasupersymmetry for our system. 


\section{Conclusion}

In this paper, the Fork space structure of a system consisting of one mode parabose and one mode parafermi degree of freedom with the same parastatistics order $p$ is investigated. Since the parabosons neither conmmute nor anticonmmute with the parafermions, the Fork space of our parasystem is more complicated than one of the ordinary bose and fermi systems, or in other words, the Fork space of our parasystem is not simply a direct product of parabose subspace and parafermi subspace. It is fortunately that there is a double degeneracy in the subspace $(m, n)$ which insures that the number of even-parafermion states is exactly the same as the number of odd-parafermion states. Based on this, the unbroken supersymmetry is established for our parasystem. An interesting task is to generalize the result of this paper to more complicated parasupersymmetric systems, and work on this direction is in progress.

\section{References}

[1] H.S.Green, Phys.Rev. 90 (1953) 270.

[2] F.Wilczek, Fractional Statistics and Anyon Superconductivity World Scientific, singapore, 1990.

[3] M.F.Sohnius, Phys.Rep. 128 (1985) 39.

[4] V.A.Rubakov and V.P.Spiridonov, Mod.Phys.Lett. A 3 (1988) 1337.

[5] J.Beckers and N.Debergh, Nucl.Phys. B340 (1990) 767.

[6] O.W.Greenberg and A.M.L.Messiah, Phys.Rev. B 138 (1965) 1155.

[7] S.N.Biswas and S.K.Soni, J.Math.Phys 29 (1988) 16.

[8] Y.Ohnuki and S.Kamefuchi, Quantum Field Theory and Parastatistics (Univ. Press of Tokyo, 1982).

[9] J.Wess and J.Bagger, Supersymmetry and Supergravity (Princeton, 1983).

[10] E.Witten, Nucl.Phys. B188 (1981) 513 and B202 (1982) 253. 